Acta Cryst. (2002). A58 (Supplement), C371

\section{ANALYSIS OF AN IONIC NETWORK WITHIN A PROTEIN OLIGOMER AND ENGENEERING TOWARDS HIGHER THERMOSTABILITY}

B.Dalhus ${ }^{1}$ A.Bjørk ${ }^{2}$ D.Mantzillas ${ }^{3}$ V.Eijsink ${ }^{4}$ R.Sirevåg ${ }^{2}$

${ }^{1}$ Department of Chemistry, University of Oslo, PO Box 1033, Blindern, N0315 Oslo, Norway. ${ }^{2}$ Department of Biology, University of Oslo, PO Box 1066, Blindern, N-0316 Oslo, Norway. ${ }^{3}$ Department of Biochemistry, University of Oslo, PO Box 1041, Blindern, N-0316 Oslo, Norway. ${ }^{4}$ Department of Chemistry and Biotechnology, Agricultural University of Norway, PO Box 5040, N-1432 Ås, Norway

Both from a fundamental and biotechnological point of view, there is considerable interest in thermostable enzymes. Enzymes from thermopcilic organisms are often in a higher oligomeric state than their mesophilic conterparts. The relative thermal stability of tetrameric malate dehydrogenase (MDH) from Chloroflexus aurantiacus (ca-MDH), Chlorobium tepidum (ct-MDH) and Chlorobium vibrioforme $(c v-\mathrm{MDH})$ can be explained by comparison of molecular interactions across the oligomeric interfaces, as revealed in the crystal structures of these proteins. ${ }^{1}$ The main differences include a conspicuous network of electrostatic dimer-dimer interactions in the most temperature resistant $c a-\mathrm{MDH}$. The contribution of the dimer-dimer interface in $c a-\mathrm{MDH}$ to thermal stability was further analyzed by studying the effects of engeneering this network of electrostatic dimer-dimer interactions. Thermal stability was strongly affected by the applied mutations, with effects of single mutations on apparent melting temperature ranging from $-12^{\circ} \mathrm{C}$ to $+24^{\circ} \mathrm{C}$. Thus, the oligomer stability can be drastically increased by optimization of the interaction network. This study provides one of a very few examples that underline the importance of multimerization by actually showing that, indeed, oligomeric enzymes may be dramatically stabilized by engeneering the multimerization interface.

REFERENCE

1. Structural Basis for Thermophilic Protein Stability: Structures of

Thermophilic and Mesophilic Malate Dehydrogenases. Dalhus, B.,

Sarinen, M., Sauer, U. H., Eklund, P., Johansson, K., Karlsson, A.,

Ramaswamy, S., Bjørk, A., Synstad, B., Naterstad, K., Sirevåg, R.

\& Eklund, H. (2002). J. Mol. Biol. In press.

Acta Cryst. (2002). A58 (Supplement), C371

THERMAL RELAXATION OF NANO-ISLAND ON SI(100)-2X1 SURFACE STUDIED BY KINETIC MONTE CARLO SIMULATION

\section{$\underline{\text { S. Toyoshima }}{ }^{1}$ and T. Kawamura ${ }^{1}$ A. Ichimiya $^{2}$}

${ }^{1}$ Dept of Math and Phys, Yamanashi University, Kofu, Yamanashi 400-8510, Japan ${ }^{2}$ Dept. of Quantum Engineering, Nagoya University, Nagoya 464-8603, Japan

Thermal relaxation plays an important role in formation of a crystallographic structure on a surface. for fabrication of nanostructure on a surface, it is necessary to control thermal relaxation process and form a self-assembled structure under an optimized condition. It is thus important to understand thermal relaxation from a microscopic point of view.

We studied the decay process of nano-island formed on $\mathrm{Si}(100)-2 \times 1$ surface by a kinetic Monte Carlo simulation. The corresponding experiment was reported by Ichimiya et al. (Surf. Sci. 493(2001)555). The decay was observed in the temperature range between $650 \mathrm{~K}$ and $800 \mathrm{~K}$ by scanning tunneling microscopy. As the system of simulation, we used a vicinal surface with $100 \times 148$ atoms on a terrace, where a 3D nano-island or a $2 \mathrm{D}$ island is formed. The periodic boundary condition was adopted. The decay is governed a mass transport of adatoms from the island to step edges of the substrate surface. The 3D island decays in a layer-by-layer fashion from the top as an adatom descends a step from one layer to the lower layer. In contrast, the 2D island decays by adatom diffusion from its edges to step edges in the substrate layers. The diffusion process is mediated by vacancies formed on the terrace by hopping of another atom from it. It is filled with an adatom diffusing on the terrace. The vacancy mediated diffusion is quite important for basic understanding of diffusion process and for fabrication of nanostructures in application.
Acta Cryst. (2002). A58 (Supplement), C371

RHEED STANDING WAVE METHOD

\section{$\underline{\text { T. Kawamura }}{ }^{1}$ P.A. Maksym ${ }^{2}$}

${ }^{1}$ Department of Mathematics and Physics, Yamanashi University, Kofu, Yamanashi 400-8510, Japan ${ }^{2}$ Department of Physics and Astronomy, University of Leicester, University Load, Leicester LE1 7RH, UK

Feasibility of RHEED standing wave method is studied for structural determination of adsorbates on $\mathrm{Si}(100)$ surface based on a multiple scattering theory. for the quantitative analysis, we calculate RHEED wave function based on the theory to show the formation of standing wave outside the crystal surface. As an example of adsorbate, we put Ge atoms on the surface. The wave function after deposition of $\mathrm{Ge}$ atoms does not change much as far as the amount of $\mathrm{Ge}$ atoms is less than $15 \%$, regardless of the adsorbed site. If the coverage of $\mathrm{Ge}$ is more than $20 \%$, not only the wave function outside the crystal changes appreciably but also that inside the crystal is modified. This indicates that a simple analysis of adsorbate site as in X-ray standing wave method is applicable to low coverage state of adsorption or growth on a crystal. If the amount of adsorbate is more than a critical value, however, an analysis based on a multiple scattering theory is needed.

The node or antinode positions of the standing wave change when the incident angle of the beam changes. It is a very advantage of this method that the site of adsorbate can be determined by continuously varying the incident angle as in the same way of measuring RHEED rocking curves. The resolution of a site determination is higher than the X-ray standing wave method, because the number of nodes in a unit length can be larger due to a multiple scattering effect.

Acta Cryst. (2002). A58 (Supplement), C371

DEBYE-WALLER FACTORS OF SI(111) SQRT\{3\} TIMES SQRT\{3\}-AG

$\underline{\text { T.Takahashi H. Tajiri, K. Sumitani S. Nakatani }}$

Institute for Solid State Physics, University of Tokyo 5-1-5 Kashiwanoha Kashiwa Chiba 277-8581 Japan

The HCT (Honeycomb Chained Triangle) model[1] is widely accepted as a structure model for the $\mathrm{Si}(111) \operatorname{sqrt}\{3\}$ times sqrt $\{3\}$-Ag surface. A new model, however, called IET (InEquivalent Triangle) model has been proposed by recent studies on the surface using scanning-tunneling microscopy at low temperatures and first principles calculations[2]. The IET model is characterized by a small amount of rotation of the Ag triangle in HCT model around its center, and the symmetry of the surface is lowered from p31m to p3. Thus the surface structure attracts renewed interests. In the present work we compare X-ray diffraction data obtained at room temperature[1] with both the HCT and IET models using anisotropic Debye-Waller factors in the least squares fitting analysis, in contrast to isotropic Debye-Waller factors in the previous analysis. The analysis indicates the experimental result is well explained by the HCT model with strong anisotropic thermal vibrations.

[1] T. Takahashi et al., Jpn. J. Appl. Phys. 27 (1988) L753, and Surf. Sci. 282 (1993) 17.

[2] H. Aizawa et al., Surf. Sci. 429 (1999) L509. 